\title{
Nutritional status in the era of target therapy: poor nutrition is a prognostic factor in non-small cell lung cancer with activating epidermal growth factor receptor mutations
}

\author{
Sehhoon Park ${ }^{1,}$, , Seongyeol Park ${ }^{1,}$, Se-Hoon Lee ${ }^{1}$, Beomseok Suh ${ }^{2}$, Bhumsuk Keam ${ }^{1}$, Tae Min Kim, \\ Dong-Wan Kim ${ }^{1}$, Young Whan Kim ${ }^{1}$, and Dae Seog $\mathrm{Heo}^{1}$
}

Departments of ${ }^{1}$ Internal Medicine and ${ }^{2}$ Family Medicine and Health Promotion Center, Seoul National University Hospital, Seoul, Korea

Received: March 9, 2015

Revised : May 15, 2015

Accepted: May 28, 2015

Correspondence to

Se-Hoon Lee, M.D.

Department of Internal Medicine, Seoul National University

Hospital, 101 Daehak-ro,

Jongno-gu, Seoul 03080, Korea

Tel: +82-2-2072-2199

Fax: $+82-2-762-9662$

E-mail: sehoon.lee119@gmail.com

*These authors contributed equally to this work.
Background/Aims: Pretreatment nutritional status is an important prognostic factor in patients treated with conventional cytotoxic chemotherapy. In the era of target therapies, its value is overlooked and has not been investigated. The aim of our study is to evaluate the value of nutritional status in targeted therapy.

Methods: A total of 2012 patients with non-small cell lung cancer (NSCLC) were reviewed and 630 patients with activating epidermal growth factor receptor (EGFR) mutation treated with EGFR tyrosine kinase inhibitor (TKI) were enrolled for the final analysis. Anemia, body mass index (BMI), and prognostic nutritional index (PNI) were considered as nutritional factors. Hazard ratio (HR), progression-free survival (PFS) and overall survival (OS) for each group were calculated by Cox proportional analysis. In addition, scores were applied for each category and the sum of scores was used for survival analysis.

Results: In univariable analysis, anemia (HR, 1.29; $p=0.015)$, BMI lower than 18.5 (HR, 1.98; $p=0.002$ ), and PNI lower than 45 (HR, 1.57; $p<0.001)$ were poor prognostic factors for PFS. Among them, BMI and PNI were independent in multi-variable analysis. All of these were also significant prognostic values for OS. The higher the sum of scores, the poorer PFS and OS were observed.

Conclusions: Pretreatment nutritional status is a prognostic marker in NSCLC patients treated with EGFR TKI. Hence, baseline nutritional status should be more carefully evaluated and adequate nutrition should be supplied to these patients.

Keywords: Nutrition status; Carcinoma, non-small-cell lung; Receptor; Epidermal growth factor; Epidermal growth factor receptor tyrosine kinase inhibitor

\section{INTRODUCTION}

More than $50 \%$ of patients with advanced cancer are diagnosed as having malnutrition with high nutritional requirement due to altered metabolic rate $[1,2]$. More- over nearly $20 \%$ of cancer patients are dying of malnutrition which is induced from tumor or treatment induced malnutrition [3]. In addition, patients under malnutrition have high risk of poor prognosis and treatment outcomes $[4,5]$. 
There have been several attempts to evaluate the patients' nutritional status before treatment and its usefulness as a prognostic marker was studied [6-8]. Among markers evaluated in previous studies, anemia [9], body mass index (BMI) [10], and prognostic nutritional index (PNI) [11] were markers which could be easily calculated from baseline laboratory exam. These markers also had strong correlation with patients' nutritional status. In addition, their roles in predicting poor prognosis have been verified in different types of cancer [6,12-14].

For the past few decades, cytotoxic chemotherapy has been the treatment of choice for patients with advanced cancer. Because of the side effects, selecting a patient tolerable for conventional chemotherapy had become greatly important before initiation of treatment [15]. In the era of molecular target therapy, small molecules such as tyrosine kinase inhibitor (TKI) demonstrated promising outcome with minimal side effects compared to conventional treatment [16]. Main treatment option of cancer with target mutation was changed from conventional chemotherapy to small molecular agents suitable for genetic profiles of tumor [17]. Now days, many patients who have not been candidates for treatment due to poor nutritional status are receiving cancer treatment using targeted agents [18].

Although nutritional status is a known prognostic marker for survival and treatment outcome of patients treated with cytotoxic chemotherapy, there has been no study conducted in patients treated with small molecular agents. We investigated the prognostic value of nutritional status in non-small cell lung cancer (NSCLC) patients with activating epidermal growth factor receptor (EGFR) mutations treated with EGFR TKI.

\section{METHODS}

\section{Study population}

A total of 2012 NSCLC patients who were treated with gefitinib or erlotinib from July 2002 to September 2014 in Seoul National University Hospital (SNUH) were reviewed. Among them, patients with confirmed activating EGFR mutation (either exon 19 deletion, or exon 21 point mutation [L858R or L861Q] by either direct DNA sequencing method or peptide nucleic acid clamping method), available pretreatment laboratory values, and physical measurement information were included. Finally, 630 patients were enrolled for the evaluation. The protocol of this study was reviewed by the institutional review board of SNUH (SNUH IRB No. 1409-143-612) and conducted in accordance with the World Medical Association's Declaration of Helsinki.

\section{Data collection}

Medical history including pathology reports, laboratory values, imaging data, and physical measurement values were collected from medical records of individual patients. Pretreatment complete blood cell count including lymphocyte proportion and serum albumin level was retrieved for each patient. Response to treatment was evaluated using computed tomography images and described by Response Evaluation Criteria in Solid Tumor criteria.

Three nutritional factors were defined as below. Anemia was categorized by serum hemoglobin level under $13 \mathrm{~g} / \mathrm{dL}$ for males and $12 \mathrm{~g} / \mathrm{dL}$ for females. BMI was categorized into three groups: BMI less than 18.5, 18.5 to 25 , and more than 25. PNI was calculated by the following formula: $10 \times$ serum albumin level $(\mathrm{g} / \mathrm{dL})+0.005 \times \mathrm{ab}$ solute lymphocyte count (number $/ \mathrm{mm}^{2}$ )

Death date of patients was collected from civil registry of South Korea, and progression-free survival (PFS) and overall survival (OS) were calculated.

\section{Statistical analysis}

Hazard ratio (HR) for each factor was calculated by Cox-regression proportional hazard model and median PFS was calculated by Kaplan-Meier analysis. Multivariable analysis was performed using factors satisfying $p$ values less than 0.05 at univariable analysis. PFS was defined as the period from the date of starting EGFR TKI to the date of cancer progression or all-cause mortality. OS was defined as the period from the date of starting EGFR TKI to the date of all-cause mortality.

Scoring system were created by conventional categorization and applied to the each nutritional factor. Higher scores were applied to categories with shorter median PFS. Score 2 was applied to the category with BMI less than 18.5 , score 1 for 18.5 to 25 , score o for more than 25 . Score 1 was also applied to patients with anemia, PNI less than 45 , and score $\mathrm{o}$ for without. The sum of scores was used for survival analysis. 
A $p$ value less than 0.05 was used as the cutoff value for statistical significance. Stata version 12.1 (Stata Co., College Station, TX, USA) was used for the statistical analysis and GraphPad Prism 5 (GraphPad Software, La Jolla, CA, USA) was used for image production.

\section{RESULTS}

\section{Characteristics of study population}

Among the 630 patients, there were 236 male (37.5\%) and 394 female (62.5\%). Pathologic type was adenocarcinoma in 588 patients (93.3\%), and $536(85.1 \%)$ have o or 1 of Eastern Cooperative Oncology Group performance status (ECOG PS). Gefitinib was used as TKI in 573 patients (91.0\%) and the other 57 (9.0\%) used erlotinib. There were 372 patients of anemia (43.2\%), 45 of BMI (7.1\%) lower than 18.5, and 177 of PNI (28.1\%) lower than 45 (Table 1).

\section{Hazard ratio of each nutritional variables}

In univariable analysis, the HR for PFS in anemia was 1.29 (95\% confidence interval [CI], 1.05 to $1.58 ; p=0.015$ ), BMI ranged from 18.5 to 25 was 1.36 (95\% CI, 1.04 to 1.78 ; $p=0.024$ ), BMI lower than 18.5 was 1.98 ( $95 \% \mathrm{CI}, 1.28$ to 3.06; $p=0.002$ ), and PNI lower than 45 was 1.57 (95\% CI, 1.26 to $1.96 ; p<0.001)$. Multivariable analysis was performed with other variables such as age at diagnosis, ECOG PS, and treatment line of TKI. In the analysis, BMI ranged from 18.5 to 25 (adjusted HR [aHR], 1.31; 95\% CI, 1.00 to 1.71; $p=0.047)$, BMI lower than 18.5 (aHR, 1.62; 95\% CI, 1.04 to $2.52 ; p=0.033)$, and PNI lower than 45 (aHR, 1.48; 95\% CI, 1.18 to 1.85 ; $p=0.001$ ) were independent factors for poor PFS, but anemia (aHR, 1.17; 95\% CI, 0.95 to $1.45 ; p=0.132$ ) lost its statistical significance.

Same approach was applied for the OS. The HR for death in anemia was 1.51 (95\% CI, 1.19 to $1.93 ; p=0.001$ ), BMI lower than 18.5 was 2.52 (95\% CI, 1.46 to 4.34 ; $p=$ 0.001), and PNI lower than 45 was 1.90 ( $95 \%$ CI, 1.47 to $2.45 ; p<0.001$ ). In multivariable analysis, all of three factors were independently significant. Detailed outcomes are shown in Table 2, Fig. 3.

\section{Actuarial difference in progression-free survival and overall survival}

In our study, all of three factors related to nutritional status have proved useful as prognostic factors for PFS
Table 1. Baseline characteristics of patients $(n=630)$

\begin{tabular}{|c|c|}
\hline Variable & No. (\%) \\
\hline Age, yr, median (range) & $64(31-91)$ \\
\hline \multicolumn{2}{|l|}{ Sex } \\
\hline Male & $236(37.5)$ \\
\hline Female & $394(62.5)$ \\
\hline \multicolumn{2}{|l|}{ Smoking } \\
\hline Current & $186(29.5)$ \\
\hline Non-smoker & $422(67.0)$ \\
\hline Not available & $22(3.5)$ \\
\hline \multicolumn{2}{|l|}{ TKI as } \\
\hline 1st line & $369(58.6)$ \\
\hline 2nd line & $235(37 \cdot 3)$ \\
\hline$>3$ rd line & $26(4.1)$ \\
\hline \multicolumn{2}{|l|}{ Pathology } \\
\hline ADC & $588(93 \cdot 3)$ \\
\hline $\mathrm{SqCC}$ & $8(1.3)$ \\
\hline Other & $34(5 \cdot 4)$ \\
\hline \multicolumn{2}{|l|}{ EGFR MT } \\
\hline Exon 19 & $367(58.3)$ \\
\hline Exon 21 & $263(41.8)$ \\
\hline \multicolumn{2}{|l|}{ TKI type } \\
\hline Gefitinib & $573(91.0)$ \\
\hline Erlotinib & $57(9.0)$ \\
\hline \multicolumn{2}{|l|}{ ECOG PS } \\
\hline $0 \& 1$ & $536(85.1)$ \\
\hline $2,3 \& 4$ & $94(14 \cdot 9)$ \\
\hline \multicolumn{2}{|l|}{ Best response } \\
\hline CR & $14(2.2)$ \\
\hline PR & $444(70.5)$ \\
\hline $\mathrm{SD}$ & $113(17 \cdot 9)$ \\
\hline $\mathrm{PD}$ & $42(6.7)$ \\
\hline Not available & $17(2.7)$ \\
\hline Anemia & $372(43.2)$ \\
\hline \multicolumn{2}{|l|}{ BMI } \\
\hline $25 \leq$ & $133(21.1)$ \\
\hline $18.5 \leq \mathrm{BMI}<25$ & $45^{2}(71.8)$ \\
\hline$<18.5$ & $45(7.1)$ \\
\hline $\mathrm{PNI}<45$ & $177(28.1)$ \\
\hline
\end{tabular}

TKI, tyrosine kinase inhibitor; ADC, adenocarcinoma; SqCC, squamous cell carcinoma; EGFR, epidermal growth factor receptor; MT, mutation; ECOG PS, Eastern Cooperative Oncology Group performance score; CR, complete response; $\mathrm{PR}$, partial response; $\mathrm{SD}$, stable disease; $\mathrm{PD}$, progressive disease; BMI, body mass index; PNI, prognostic nutritional index. 


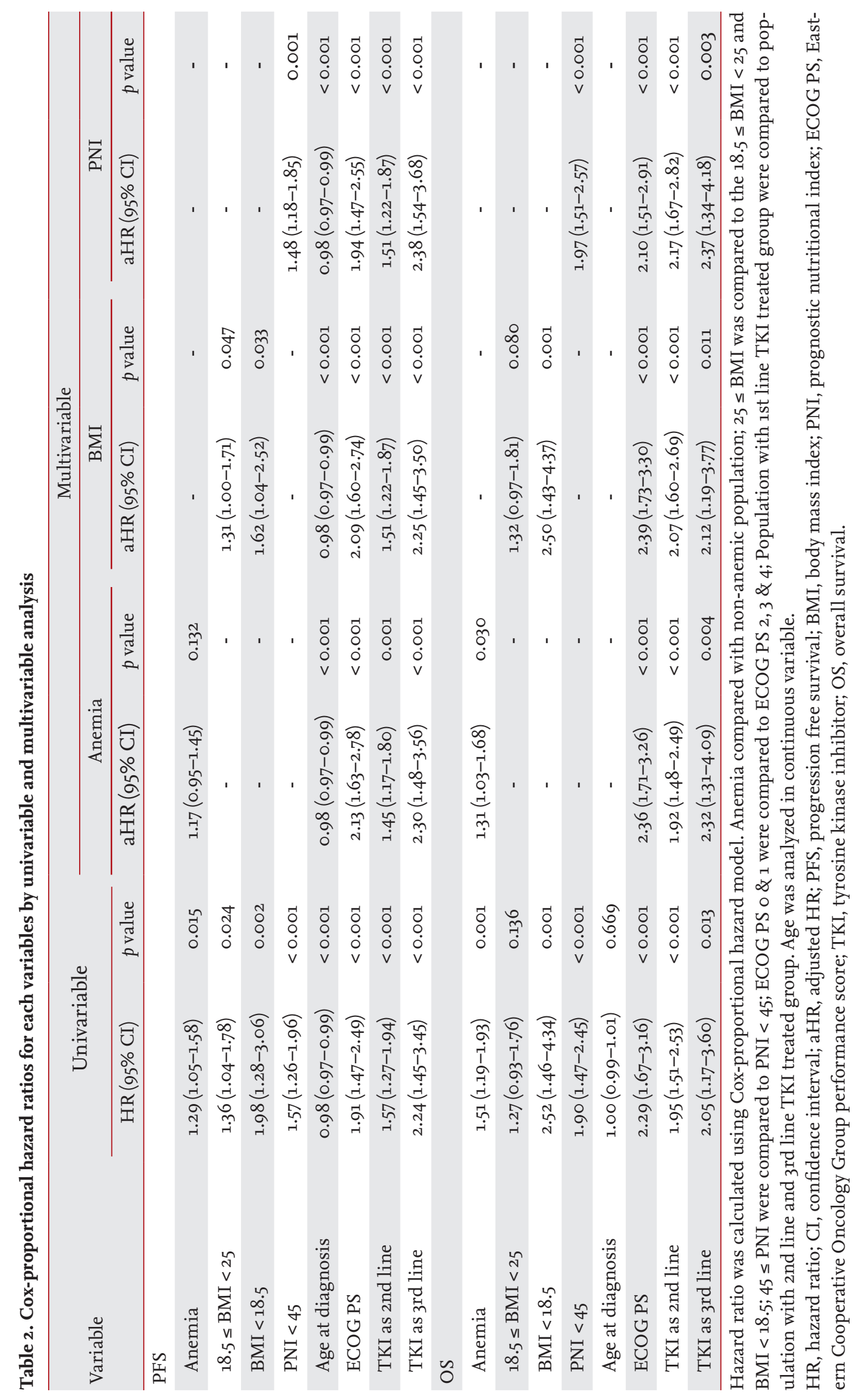



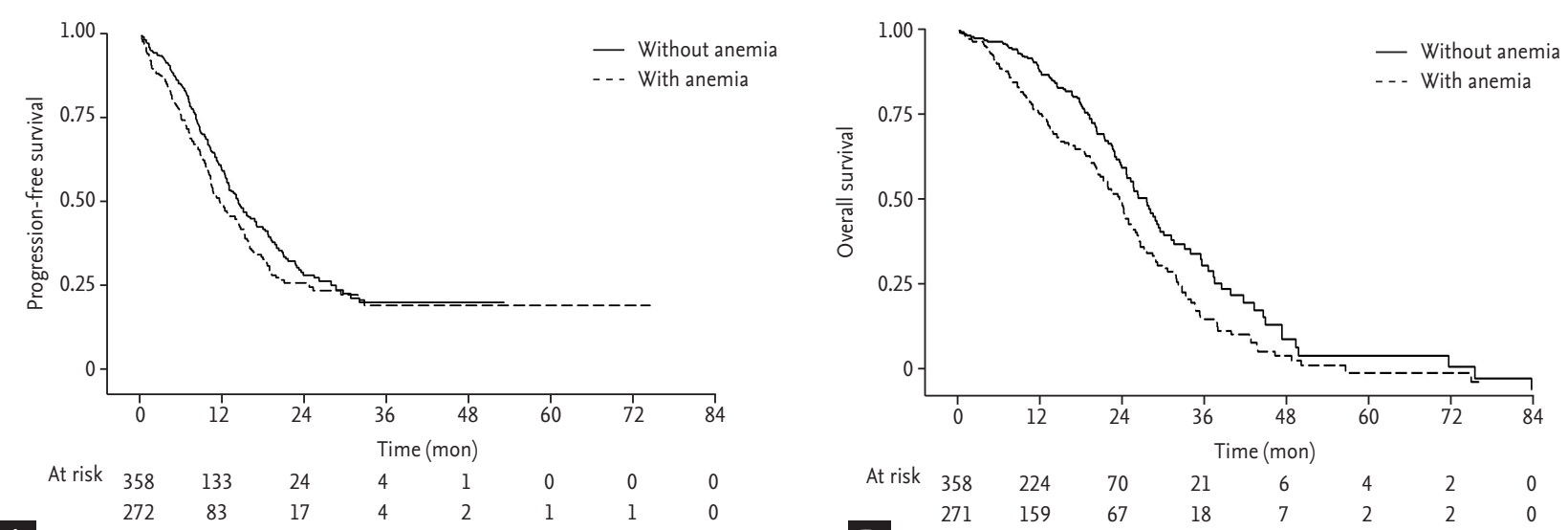

A

$\begin{array}{ccccccccc}\text { At risk } & 358 & 133 & 24 & 4 & 1 & 0 & 0 & 0 \\ & 272 & 83 & 17 & 4 & 2 & 1 & 1 & 0\end{array}$

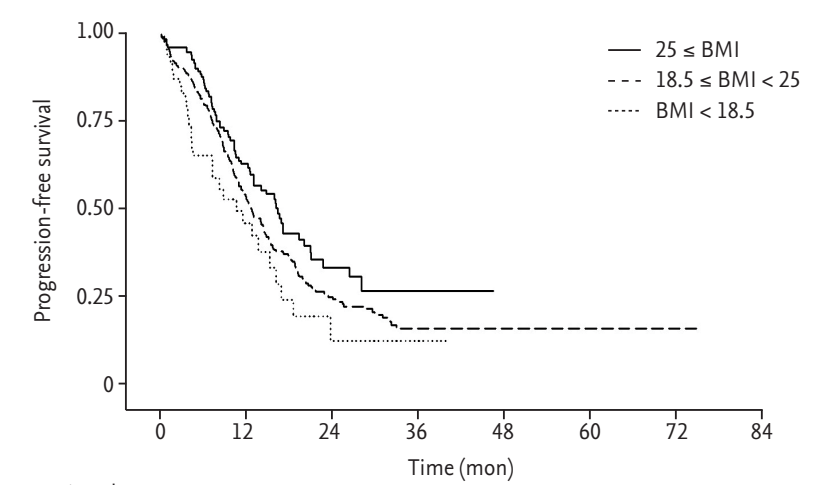

D

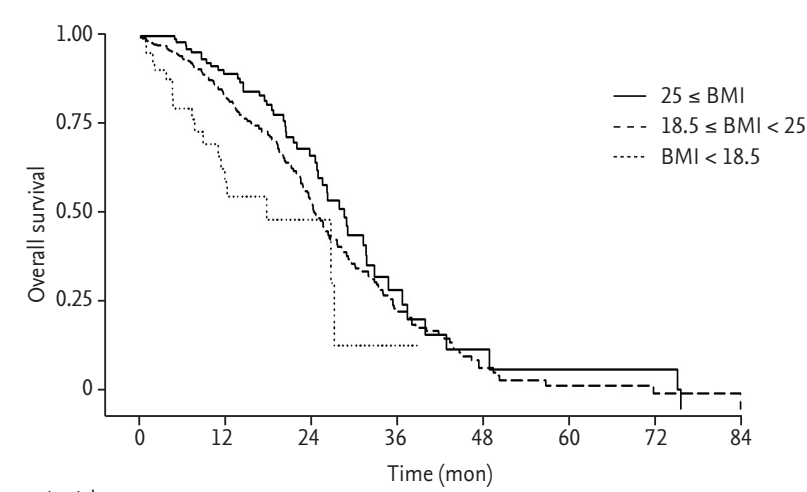

B

$\begin{array}{ccccccccc}\text { At risk } & 133 & 56 & 11 & 1 & 0 & 0 & 0 & 0 \\ & 452 & 149 & 29 & 6 & 3 & 1 & 1 & 0 \\ & 11 & 1 & 1 & 0 & 0 & 0 & 0\end{array}$

$\begin{array}{ccccccccc}\text { At risk } & 133 & 87 & 35 & 8 & 3 & 2 & 2 & 0 \\ & 451 & 279 & 99 & 30 & 10 & 4 & 2 & 0 \\ \text { E } & 45 & 17 & 3 & 1 & 0 & 0 & 0 & 0\end{array}$

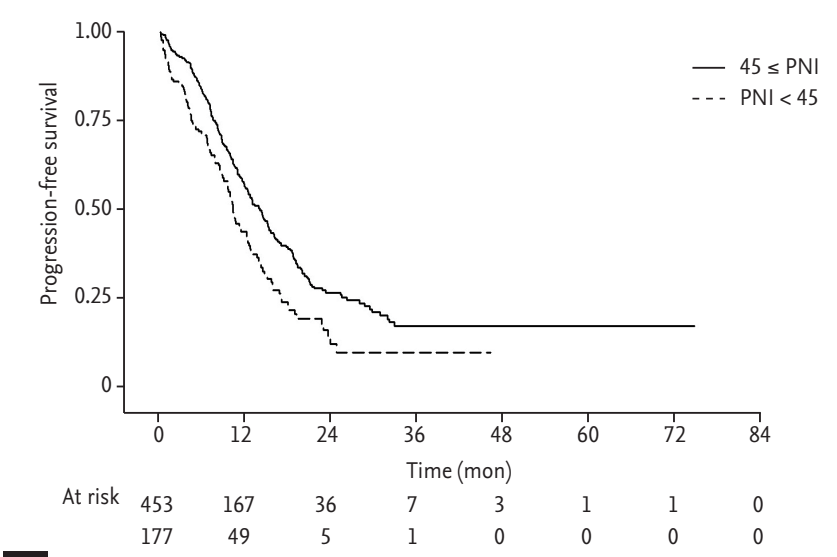

C

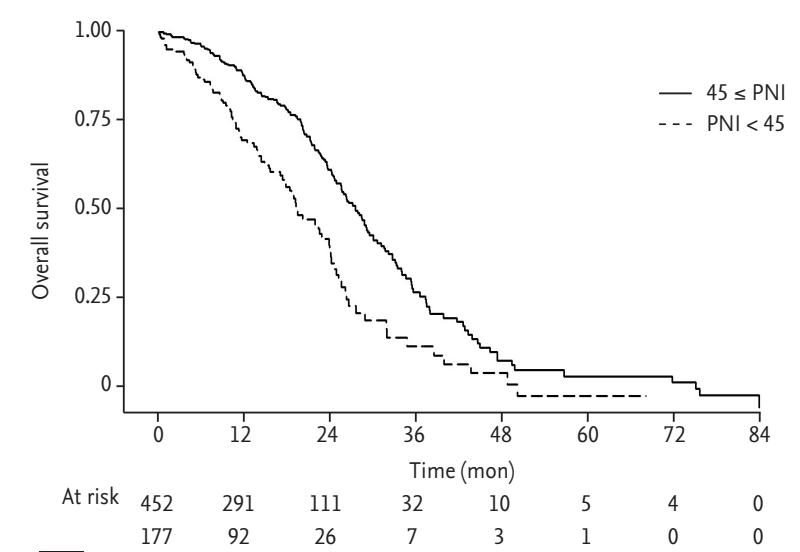

Figure 1. Kaplan-Meier curves of progression free survival (PFS) and overall survival (OS) by nutritional variables. (A) PFS curve plotted by group with anemia and group without anemia. (B) PFS curve plotted by group distributed by body mass index (BMI) value of 18.5 and 25. (C) PFS curve plotted by group distributed by prognostic nutritional index (PNI) value of 45 . (D) OS curve plotted by group with anemia and group without anemia. (E) OS curve plotted by group distributed by BMI value of 18.5 and 25. (F) OS curve plotted by group distributed by PNI value of 45 .

and OS with EGFR TKI treatment. There were decreased median PFS of 2.3 months in anemia, 3.9 in BMI ranged from 18.5 to 25, 7.1 in BMI lower than 18.5, and 3.0 in PNI lower than 45. Similar tendency was observed for OS ex- 
Table 3. Scoring system based on patients' nutritional status and distribution of patients by sum of score

\begin{tabular}{|c|c|c|c|c|}
\hline Category & No. (\%) & Score & Median PFS (95\% CI) & Median OS (95\% CI) \\
\hline \multicolumn{5}{|l|}{ Anemia } \\
\hline No & - & 0 & $12.6(11.9-14.6)$ & $28.1(25.6-31.5)$ \\
\hline Yes & - & 1 & $10.3(9.4-12.1)$ & $24.2(21.8-26.3)$ \\
\hline \multicolumn{5}{|l|}{ BMI } \\
\hline $25 \leq \mathrm{BMI}$ & - & o & $15.6(11.8-16.6)$ & $28.8(24.9-32.8)$ \\
\hline $18.5 \leq \mathrm{BMI}<25$ & - & 1 & $11.7(10.6-12.7)$ & $25.6(23.8-27.6)$ \\
\hline $\mathrm{BMI}<18.5$ & - & 2 & $8.5(4.2-14.8)$ & $26.7(10.8-27.1)$ \\
\hline \multicolumn{5}{|l|}{ PNI } \\
\hline $45 \leq \mathrm{PNI}$ & - & o & $13.0(11.7-14.8)$ & $28.5(26.2-31.5)$ \\
\hline $\mathrm{PNI}<45$ & - & 1 & $10.0(8.5-11.8)$ & $21.9(17.8-24.1)$ \\
\hline \multicolumn{5}{|l|}{ Sum of score } \\
\hline o & $73(11.6)$ & & $19.5(12.1-27.3)$ & $29.0(24.7-37.3)$ \\
\hline 1 & $243(38.6)$ & & $13.9(11.5-15.4)$ & $29.5(25.7-35.5)$ \\
\hline 2 & $202(32.0)$ & & $11.2(9.7-12.7)$ & $24.9(22.8-27.5)$ \\
\hline 3 & $104(16.5)$ & & $9 \cdot 3(7.0-11.8)$ & $17.6(12.5-23.9)$ \\
\hline 4 & $8(1.3)$ & & $7.0(2.6-)$ & $11.2(7.2-)$ \\
\hline
\end{tabular}

PFS, progression free survival; CI, confidence interval; OS, overall survival; BMI, body mass index; PNI, prognostic nutritional index.

cept the subjects with BMI lower than 18.5 (median OS, 26.7 months), as compared with BMI ranged from 18.5 to 25 (median OS, 25.6 months). Detailed data is described in Table 3, and Kaplan-Meier curves for each factor is plotted in Fig. 1.

\section{Risk analysis using scoring system}

Each factor was scored by conventional distribution. The sum of scores was considered as a surrogated marker of patients' nutritional status and survival analysis was done. The subjects with score o was considered as the best nutritional status group, and the subjects with score 4 was considered as the poorest nutritional status group. The subjects with the higher score showed the shorter median PFS and OS as shown in Table 3, Fig. 2. In the same manner, the subjects with high score showed increasing tendency of HR compared to the subjects with score $\mathrm{o}$.

\section{DISCUSSION}

Pretreatment nutritional status is an important factor to be considered when planning cancer treatment. Ane- mia, low BMI, and low PNI are factors which can be easily calculated and represent patients' nutritional status. Our study showed usefulness of these factors as prognostic markers in patients treated with TKI. Moreover, effectiveness of the simple scoring system representing personal nutritional status was suggested.

There are two important contributing factors that are related to malnutrition in cancer patients. One is increased metabolic rates and the other is reduced food intake due to cancer or treatment related symptoms. Increased energy consumption is augmented by cytokines produced by tumor and they activate false adaptive system in the brain and alter the homeostasis of energy storage. As a consequence, muscle wasting and lipolysis proceed and more calories are needed to prevent the catabolism. As the disease progress, patients are more likely to be functionally impaired and show intolerance to anticancer drug [19]. The other factor, reduced food intake, can be managed with active nutritional intervention, appetite stimulants, and antiemetics [20]. Sometimes, however, there are limitations of nutritional intervention in gastrointestinal cancer with anatomical obstruction or head and neck cancer with dysphagia. For these reasons, baseline nutritional status is import- 

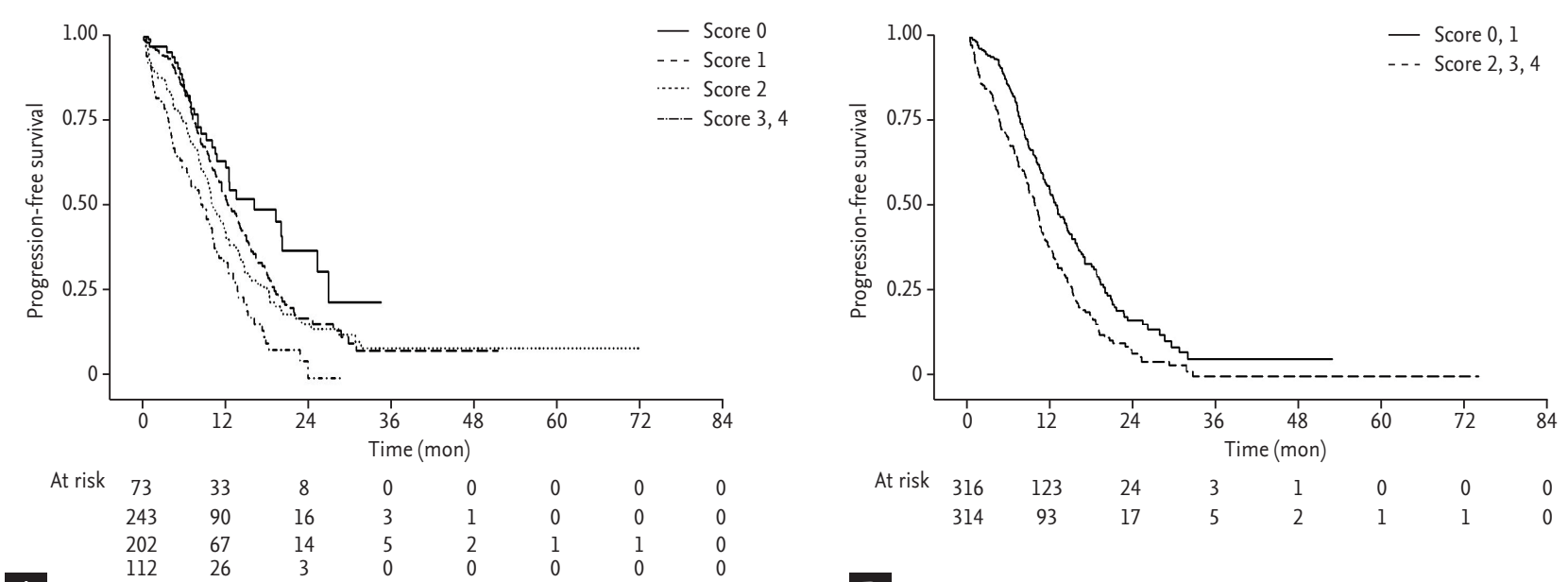

A

\section{B}
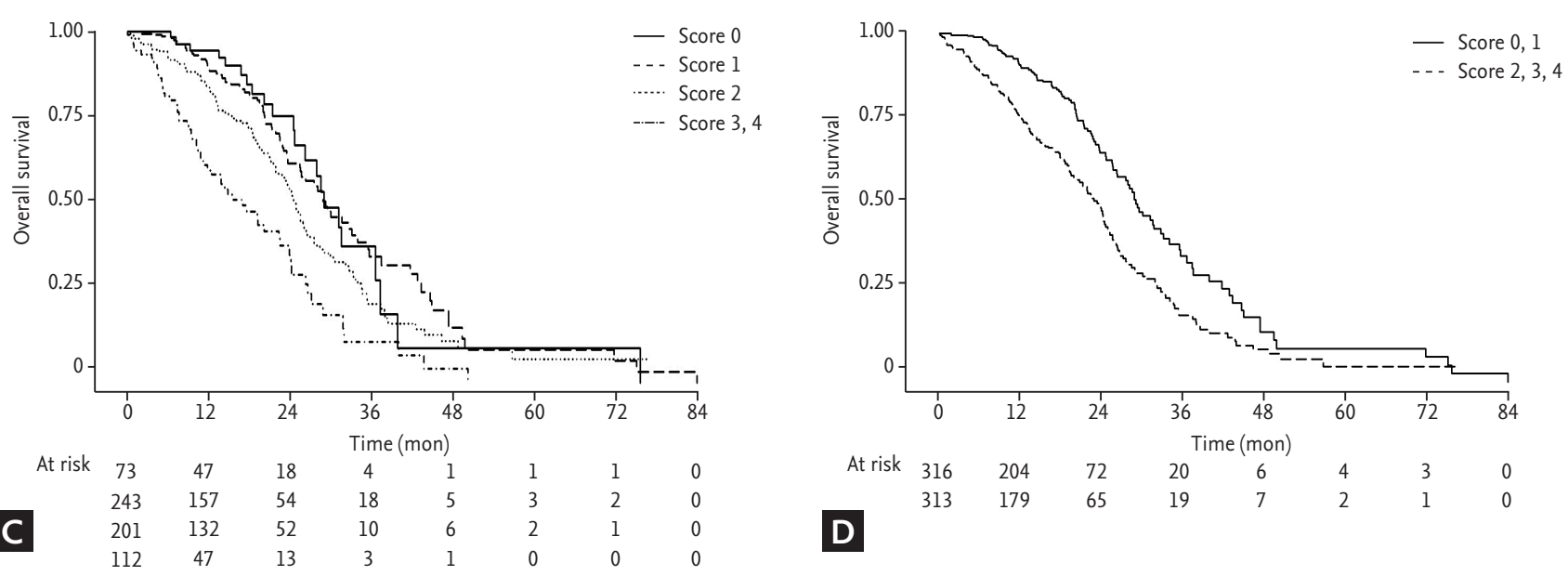

Figure 2. Kaplan-Meier curves of progression free survival (PFS) and overall survival (OS) by sum of score and by group with sum of score 0,1 and group with 2, 3, 4. (A) PFS curve plotted by sum of score. (B) PFS curve plotted by group with sum of score $\mathrm{O}, 1$ and group with $2,3,4$. (C) OS curve plotted by sum of score. (D) OS curve plotted by group with sum of score 0,1 and group with 2, 3, 4 .

ant and malnutrition can be a sign of poor prognosis with less treatment options.

Pretreatment risk assessment is routinely performed by complete history taking including comorbidities and performance status. Among the indices, performance status graded by ECOG PS [21], developed in 1982 by ECOG, is the most commonly used for prognostic index and has been proved its clinical significance as a prognostic marker [22]. In addition to the ECOG PS, nutritional status is emerging as an important prognostic factor $[23,24]$.

Previous data showed active oral nutritional intervention has positive effect in decreased morbidity [25] and improves quality of life [26]. Moreover, there is a report that nutritional intervention improves tolerance to chemotherapy [4]. Regarding the above information, patients with higher scores in our study should be considered as a high risk population of treatment failure, and active nutritional intervention should be considered to these patients.

Both anemia and low PNI have been demonstrated to be associated with higher risk of poor prognosis. Although no previous study was on NSCLC patients with BMI, there have been controversial results, in which obesity was reported as a risk factor for early cancer related mortality. But this can be explained by the unique pathophysiology of breast cancer in relation to sex hormones being produced more in adipose tissue of obese 

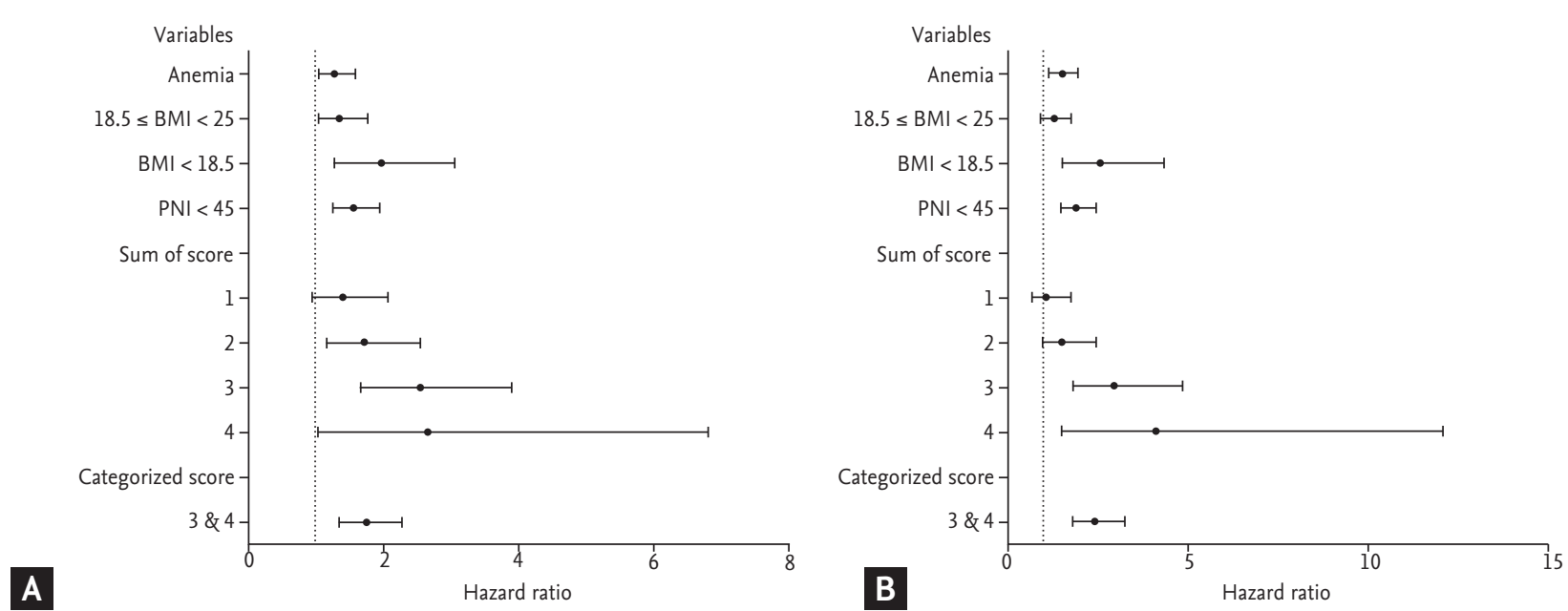

Figure 3. Forest plot of hazard ratio for progression-free survival (PFS) and overall survival. (A) Hazard ratio (HR) for progression free survival. HRs for variables are listed in Table 2. HR for PFS is as following: group with score 1 is 1.41 (95\% confidence interval [CI], 0.96 to $2.08 ; p=0.078$ ); group with score 2 is 1.72 (95\% CI, 1.17 to 2.54 ; $p=0.006$ ); group with score 3 is 2.57 (95\% CI, 1.69 to 3.90; $p<0.001$ ); group with score 4 is 2.66 (95\% CI, 1.03 to $6.82 ; p=0.043$ ); group combined with score 3 and 4 is 1.75 (95\% CI, 1.36 to 2.27 ; $p<0.001$ ). (B) HR for overall survival is as following: group with score 1 is 1.07 (95\% CI, 0.67 to 1.71 ; $p=0.786$ ); group with score 2 is 1.53 (95\% CI, 0.96 to 2.43 ; $p=0.073$ ); group with score 3 is 2.95 (95\% CI, 1.80 to 4.84 ; $p<0.001$ ); group with score 4 is 4.11 (95\% CI, 1.41 to $11.97 ; p=0.010$ ); group combined with score 3 and 4 is 2.42 (95\% CI, 1.81 to $3.24 ; p<0.001$ ).

patients [27-29]. Overall malnutrition as a risk factor in NSCLC should be understood in other ways. In the concept of risk factor paradox introduced in chronic disease, obesity showed protective effect in wasting disease, including cancer [30]. As patients with other wasting disease, our patients with high BMI showed lower incidence of adverse effects than patients with low BMI. This can be explained by time differential of competing risks [14]. Applying the time discrepancy between the groups categorized by nutrition factors, paradoxical effects of overweight are expected to be more prominent in the era of target therapy, due to prolonged survival and elongated duration of the treatment.

In our study, all of the three factors, anemia, BMI, and PNI, were significant as prognostic factors for PFS with EGFR TKI and OS. And these results maintained with the adjustment of ECOG PS. This is consistent with a previous study that demonstrated early progression of disease in cachexic patients compared to non-cachexic patients [31]. Although PS is routinely used as a means of representing overall physical condition in cancer patients, there is a shortage of evaluating nutritional status before treatment.

There are several limitations in our study. First, our study population is retrospective and does not represent the whole population treated with targeted agents.
Nonetheless, EGFR TKI is commonly used small molecules and our study population has been well controlled by validated mutation analysis. Second, the scoring system used in our study has not been verified previously. Therefore, its usefulness should be confirmed in future studies.

Now days, increased number of patients are receiving cancer treatment using targeted therapy. However, individual nutritional status has being overlooked due to minimal side effects of small molecular agents. Since our study revealed the importance of baseline nutritional status as a prognostic factor, pretreatment evaluation of nutritional status and adequate nutritional support should be emphasized even in the patients receiving targeted therapy.

\section{KEY MESSAGE}

1. In the era of targeted therapy, small molecules demonstrated promising outcome with minimal side effects compared to conventional treatment. More patients are receiving targeted agent seven if they have poor nutritional status.

2. Our study proved usefulness of non-invasive nutritional indices, anemia, body mass index, and prognostic nutritional index as prognostic 
markers in non-small cell lung cancer patients treated with epidermal growth factor receptor tyrosine kinase inhibitor.

\section{Conflict of interest}

No potential conflict of interest relevant to this article was reported.

\section{Acknowledgments}

This study was supported by a grant from the Innovative Research Institute for Cell Therapy, Korea (Ao62260).

\section{REFERENCES}

1. Argiles JM, Busquets S, Stemmler B, Lopez-Soriano FJ. Cancer cachexia: understanding the molecular basis. Nat Rev Cancer 2014;14:754-762.

2. Lieffers JR, Mourtzakis M, Hall KD, McCargar LJ, Prado $\mathrm{CM}$, Baracos VE. A viscerally driven cachexia syndrome in patients with advanced colorectal cancer: contributions of organ and tumor mass to whole-body energy demands. Am J Clin Nutr 2009;89:1173-1179.

3. Ottery FD. Cancer cachexia: prevention, early diagnosis, and management. Cancer Pract 1994;2:123-131.

4. Paccagnella A, Morassutti I, Rosti G. Nutritional intervention for improving treatment tolerance in cancer patients. Curr Opin Oncol 2011;23:322-330.

5. Correia MI, Waitzberg DL. The impact of malnutrition on morbidity, mortality, length of hospital stay and costs evaluated through a multivariate model analysis. Clin Nutr 2003;22:235-239.

6. Martin L, Birdsell L, Macdonald N, et al. Cancer cachexia in the age of obesity: skeletal muscle depletion is a powerful prognostic factor, independent of body mass index. J Clin Oncol 2013;31:1539-1547.

7. Fearon K, Strasser F, Anker SD, et al. Definition and classification of cancer cachexia: an international consensus. Lancet Oncol 2011;12:489-495.

8. Brookes GB. Nutritional status: a prognostic indicator in head and neck cancer. Otolaryngol Head Neck Surg 1985;93:69-74.

9. Mitrache C, Passweg JR, Libura J, et al. Anemia: an indicator for malnutrition in the elderly. Ann Hematol 2001;80:295-298.
10. Bailey KV, Ferro-Luzzi A. Use of body mass index of adults in assessing individual and community nutritional status. Bull World Health Organ 1995;73:673-680.

11. Onodera T, Goseki N, Kosaki G. Prognostic nutritional index in gastrointestinal surgery of malnourished cancer patients. Nihon Geka Gakkai Zasshi1984;85:1001-1005.

12. Szkandera J, Gerger A, Liegl-Atzwanger B, et al. Pre-treatment anemia is a poor prognostic factor in soft tissue sarcoma patients. PLoS One 2014;9:e107297.

13. Nozoe T, Ninomiya M, Maeda T, Matsukuma A, Nakashima H, Ezaki T. Prognostic nutritional index: a tool to predict the biological aggressiveness of gastric carcinoma. Surg Today 2010;40:440-443.

14. Kalantar-Zadeh K, Horwich TB, Oreopoulos A, et al. Risk factor paradox in wasting diseases. Curr Opin Clin Nutr Metab Care 2007;10:433-442.

15. Gridelli C, Ardizzoni A, Le Chevalier T, et al. Treatment of advanced non-small-cell lung cancer patients with ECOG performance status 2: results of an European Experts Panel. Ann Oncol 2004;15:419-426.

16. Yang JC, Hirsh V, Schuler M, et al. Symptom control and quality of life in LUX-Lung 3: a phase III study of afatinib or cisplatin/pemetrexed in patients with advanced lung adenocarcinoma with EGFR mutations. J Clin Oncol 2013;31:3342-3350.

17. Arora A, Scholar EM. Role of tyrosine kinase inhibitors in cancer therapy. J Pharmacol Exp Ther 2005;315:971-979.

18. Reck M, Popat S, Reinmuth N, et al. Metastatic nonsmall-cell lung cancer (NSCLC): ESMO Clinical Practice Guidelines for diagnosis, treatment and follow-up. Ann Oncol 2014;25 Suppl 3:iii27-iiiz9.

19. Fearon K, Arends J, Baracos V. Understanding the mechanisms and treatment options in cancer cachexia. Nat Rev Clin Oncol 2013;10:90-99.

20. Simons JP, Schols AM, Hoefnagels JM, Westerterp KR, ten Velde GP, Wouters EF. Effects of medroxyprogesterone acetate on food intake, body composition, and resting energy expenditure in patients with advanced, nonhormone-sensitive cancer: a randomized, placebo-controlled trial. Cancer 1998;82:553-560.

21. Oken MM, Creech RH, Tormey DC, et al. Toxicity and response criteria of the Eastern Cooperative Oncology Group. Am J Clin Oncol 1982;5:649-655.

22. Finkelstein DM, Ettinger DS, Ruckdeschel JC. Long-term survivors in metastatic non-small-cell lung cancer: an Eastern Cooperative Oncology Group Study. J Clin Oncol 
1986;4:702-709.

23. Cessot A, Coriat R, Mir O, et al. Nutritional status is superior to the ECOG performance status in predicting the dose-intensity of the GEMOX chemotherapy regimen in patients with advanced cancer. Nutr Cancer 2013;65:12541257.

24. Capuano G, Gentile PC, Bianciardi F, Tosti M, Palladino A, Di Palma M. Prevalence and influence of malnutrition on quality of life and performance status in patients with locally advanced head and neck cancer before treatment. Support Care Cancer 2010;18:433-437.

25. Ravasco P, Monteiro-Grillo I, Vidal PM, Camilo ME. Dietary counseling improves patient outcomes: a prospective, randomized, controlled trial in colorectal cancer patients undergoing radiotherapy. J Clin Oncol 2005;23:14311438.

26. Baldwin C, Spiro A, Ahern R, Emery PW. Oral nutritional interventions in malnourished patients with cancer: a systematic review and meta-analysis. J Natl Cancer Inst 2012;104:371-385.
27. Berclaz G, Li S, Price KN, et al. Body mass index as a prognostic feature in operable breast cancer: the International Breast Cancer Study Group experience. Ann Oncol 2004;15:875-884.

28. Dawood S, Broglio K, Gonzalez-Angulo AM, et al. Prognostic value of body mass index in locally advanced breast cancer. Clin Cancer Res 2008;14:1718-1725.

29. Key TJ, Appleby PN, Reeves GK, et al. Body mass index, serum sex hormones, and breast cancer risk in postmenopausal women. J Natl Cancer Inst 2003;95:1218-1226.

30. Halabi S, Small EJ, Vogelzang NJ. Elevated body mass index predicts for longer overall survival duration in men with metastatic hormone-refractory prostate cancer. J Clin Oncol 2005;23:2434-2435.

31. Fearon KC, Voss AC, Hustead DS; Cancer Cachexia Study Group. Definition of cancer cachexia: effect of weight loss, reduced food intake, and systemic inflammation on functional status and prognosis. Am J Clin Nutr 2006;83:1345-1350. 